\title{
Erratum
}

\section{A radio and mid-infrared survey of northern bright-rimmed clouds}

\author{
L. K. Morgan ${ }^{1,2}$, M. A. Thompson ${ }^{1}$, J. S. Urquhart ${ }^{1}$, G. J. White ${ }^{1}$, and J. Miao ${ }^{1}$ \\ 1 Centre for Astrophysics \& Planetary Science, School of Physical Sciences, University of Kent, Canterbury, Kent CT2 7NR, UK \\ 2 NRAO Green Bank Observatory, Green Bank, WV 24944, USA \\ e-mail: lmorgan@nrao.edu
}

A\&A 426, 535-545 (2004), DOI:10.1051/0004-6361:20040226

Key words. stars: formation - radio continuum: stars - ISM: clouds - ISM: molecules - HII regions - errata, addenda

We report the discovery of an error in the calculation of the electron densities and ionised gas pressures in Morgan et al. (2004). The electron density $\left(n_{\mathrm{e}}\right)$ of each IBL surrounding the clouds discussed in Morgan et al (2004) was derived using their Eq. (2).

$n_{\mathrm{e}}=122.41 \sqrt{\frac{S_{v} T_{\mathrm{e}}^{0.35} v^{0.1} \theta^{-2}}{\eta R}}$

where $S_{v}$ is the integrated radio flux in mJy, $T_{\mathrm{e}}$ is the effective electron temperature of the ionised gas in $\mathrm{K}, v$ is the frequency of the free-free emission in $\mathrm{GHz}$ and $\theta$ is the angular diameter over which the emission is integrated in arcseconds. $R$ is the radius of the cloud in pc and $\eta$ is the effective thickness of the IBL as a fraction of the cloud radius (typically $\eta \sim 0.2$ ).

Original calculations of $n_{\mathrm{e}}$ were made using incorrect values of $S_{v}$. These incorrect values had not taken beamsize and pixel size corrections into account and thus generally exaggerated values of the integrated flux. This was a computational error, the integrated fluxes presented in the paper, along with the derived ionising fluxes are correct.

Incorrectly calculated integrated flux values used in computation led to overestimations of electron density and thus ionised gas pressure. In order to correct the mistakes propagated through the work by this miscalculation the changes listed below should be noted.

1) In the abstract, two clouds have been found in which the pressure exerted by their IBLs is much greater than that measured in the internal molecular material, not three as previously stated. This discrepancy is repeated in paragraph 7 of Sect. 4.1 (p. 542) and again in paragraphs 1 and 2 of Sect. 5 (p. 543).

2) Table 2 presented in this erratum replaces that of the original work.

3) Paragraph 5 of Sect. 4.1 (p. 542) should be replaced with the following text "The IBL pressures calculated from the NVSS data range from 16.2 to $85.8 \times 10^{5} \mathrm{~cm}^{-3} \mathrm{~K}$ with a mean of $33.72 \times 10^{5} \mathrm{~cm}^{-3} \mathrm{~K}$ while the molecular line data that has

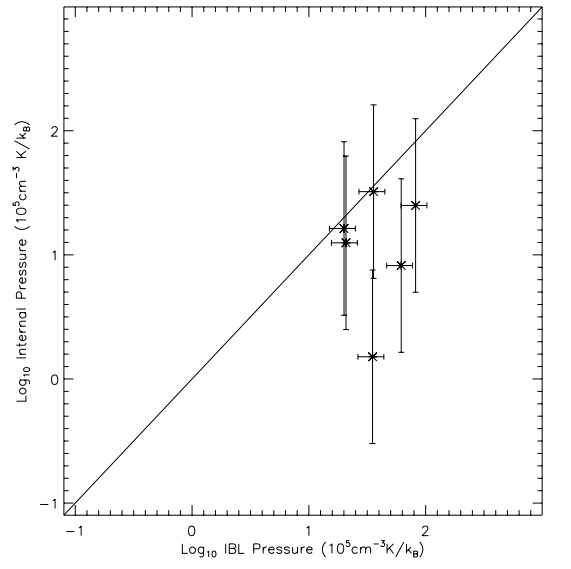

Fig. 1. Graph of internal molecular pressure versus IBL pressure. Error bars in IBL pressure represent an approximate $75 \%$ uncertainty in the assumption that $T_{\mathrm{e}}=10^{4}$. The solid line indicates pressure balance between the internal and external pressures.

been collected show a range of internal molecular pressures of $0.3-32.3 \times 10^{5} \mathrm{~cm}^{-3} \mathrm{~K}$ with a mean of $12.3 \times 10^{5} \mathrm{~cm}^{-3} \mathrm{~K}$."

4) The last paragraph of Sect. 4.1 (p. 542) should now read "The mean internal pressure of the clouds for which we have molecular line data is $12.3 \times 10^{5} \mathrm{~cm}^{-3} \mathrm{~K}$ (consistent with the results of Thompson et al. (2004b) who find an internal molecular pressure of $15 \times 10^{5} \mathrm{~cm}^{-3} \mathrm{~K}$ ), the remainder of our clouds for which we have $20 \mathrm{~cm}$ data but no molecular data have been found to have ionised gas pressures higher than this without exception. All of the clouds within our sample are therefore candidates for the possible propagation of photoionisation induced shocks into their interiors, although as this determination is based upon a global mean of internal pressure no definitive statements can be made. However, with an external pressure of $85.8 \times 10^{5} \mathrm{~cm}^{-3} \mathrm{~K}$ the BRC SFO 1 is highly likely to be underpressured with respect to their IBLs. Together with SFO 7 and SFO 36, which are the two clouds most likely to be overpressured with respect to their IBLs with IBL pressures comparable to the global mean internal pressure, these clouds 
Table 2. Values for the measured ionising flux, predicted ionising flux, the measured electron density and ionised gas pressure for type 1 radio sources detected in the survey.

\begin{tabular}{ccccc}
\hline \hline SFO Object & $\begin{array}{c}\text { Measured ionising flux } \\
\Phi\left(10^{8} \mathrm{~cm}^{-2} \mathrm{~s}^{-1}\right)\end{array}$ & $\begin{array}{c}\text { Predicted ionising flux } \\
\Phi_{P}\left(10^{8} \mathrm{~cm}^{-2} \mathrm{~s}^{-1}\right)\end{array}$ & $\begin{array}{c}\text { Electron Density } \\
n_{\mathrm{e}}\left(\mathrm{cm}^{-3}\right)\end{array}$ & $\begin{array}{c}\text { Ionised gas pressure } \\
P_{i} / k_{\mathrm{B}}\left(10^{5} \mathrm{~cm}^{-3} \mathrm{~K}\right)\end{array}$ \\
\hline 1 & 18.5 & 43.7 & 272 & 85.8 \\
2 & 2.7 & 2.1 & 84 & 26.7 \\
4 & 2.7 & 43.1 & 196 & 61.8 \\
5 & 7.5 & 61.3 & 113 & 35.8 \\
6 & 3.1 & 32.1 & 121 & 38.2 \\
7 & 3.6 & 78.7 & 51 & 16.2 \\
10 & 9.9 & 10.5 & 161 & 50.8 \\
11 & 2.3 & 10.2 & 72 & 22.8 \\
12 & 5.2 & 44.5 & 109 & 34.5 \\
13 & 3.2 & 12.4 & 63 & 20.0 \\
15 & 3.0 & 18.8 & 71 & 22.4 \\
25 & 2.2 & 27.1 & 66 & 20.8 \\
27 & 5.6 & 1.7 & 133 & 42.1 \\
30 & 22.1 & 4065.9 & 142 & 44.7 \\
31 & 8.7 & 3.5 & 151 & 37.5 \\
32 & 2.2 & 11.4 & 124 & 20.1 \\
35 & 1.2 & 6.9 & 63 & 17.2 \\
36 & 2.4 & 59.6 & 54 & 35.1 \\
37 & 2.0 & 10.2 & 111 & 24.4 \\
38 & 2.8 & 13.3 & 77 & 34.3 \\
40 & 2.5 & 8.1 & 108 & 24.4 \\
41 & 1.5 & 8.0 & 77 & 24.6 \\
42 & 1.3 & 7.3 & 78 & 27.3 \\
43 & 4.8 & 145.8 & 86 & 26.5 \\
44 & 2.1 & 4.6 & 84 &
\end{tabular}

Table 6. Ionised boundary layer pressures, internal pressures and densities of clouds observed in molecular line wavelengths. A dash indicates non-detection in the NVSS.

\begin{tabular}{cccc}
\hline \hline SFO Object & $\begin{array}{c}\text { IBL pressure } \\
\left(10^{5} \mathrm{~cm}^{-3} \mathrm{~K}\right)\end{array}$ & $\begin{array}{c}\text { Internal Pressure } \\
\left(10^{5} \mathrm{~cm}^{-3} \mathrm{~K}\right)\end{array}$ & $\begin{array}{c}\text { Internal Molecular Density } \\
\left(10^{3} \mathrm{~cm}^{-3}\right)\end{array}$ \\
\hline $4^{a}$ & 61.8 & 8.2 & 50.0 \\
$5^{b}$ & 35.8 & 32.3 & 11.0 \\
$11^{c}$ & 82.0 & 25.0 & 2.5 \\
$13^{d}$ & 20.0 & 16.3 & 2.0 \\
$16^{d}$ & - & 0.3 & 2.0 \\
$18^{d}$ & - & 7.8 & 16.6 \\
$20^{e}$ & - & 6.9 & 20.0 \\
$25^{d}$ & 20.8 & 12.5 & 7.4 \\
$37^{f}$ & 35.1 & 1.5 & 3.0 \\
\hline
\end{tabular}

${ }^{a}$ Jansen et al. (1994); ${ }^{b}$ Lefloch et al. (1997); ${ }^{c}$ Thompson et al. (2004a); ${ }^{d}$ De Vries et al. (2002); ${ }^{e}$ Cernicharo et al. (1992); ${ }^{f}$ Duvert et al. (1990)

represent good candidates for future molecular observations to accurately determine their internal pressures."

5) The end of paragraph 1 of Sect. 5 (p. 543) should now read "A comparison of the results that we have derived for SFO 5 with those of Lefloch et al. (1997) reveals a difference in derived IBL pressure of a factor of $\sim 2(32.3 / 67.6)$, this is believed to be due to the differences in observational data (frequency, integration area, etc.), we do not feel that these differences unduly affect our conclusions as the other errors inherent in our derivation are as large or larger than that arising from this beam dilution."

6) Figure 1 presented in this erratum replaces that of the original work.
7) Table 6 presented in this erratum replaces that of the original work.

\section{References}

Cernicharo, J., Bachiller, R., Duvert, G., et al. 1992, A\&A, 261, 589 De Vries, C. H., Narayanan, G., \& Snell, R. L. 2002, ApJ, 577, 798 Duvert, G., Cernicharo, J., Bachiller, R., \& Gomez-Gonzalez, J. 1990, A\&A, 233, 190

Jansen, D. J., van Dishoeck, E. F., \& Black, J. H. 1994, A\&A, 282, 605 Lefloch, B., \& Lazareff, B. 1994, A\&A, 289, 559

Lefloch, B., Lazareff, B., \& Castets, A. 1997, A\&A, 324, 249

Morgan, L. K., Thompson, M. A., Urquhart, J. S., White, G. J., \& Miao, J. 2004, A\&A, 426, 535

Morgan, L. K., Thompson, M. A., Urquhart, J. S., White, G. J., \& Miao, J. 2004, in preparation

Sugitani, K., Fukui, Y., \& Ogura, K. 1991, ApJS, 77, 59

Thompson, M. A., White, G. J., Morgan, L. K., et al. 2004a, A\&A, 414, 1017 Thompson, M. A., Urquhart, J. S., \& White, G. J. 2004b, A\&A, 415, 627 